\title{
Integrated Electric Vehicle Control by Differential Parameterization
}

\author{
Jens Hoedt and Ulrich Konigorski
}

\begin{abstract}
The design of a tracking controller for electric cars in $\mathbf{x}$-by-wire structure is presented to assign trajectories for the coordinates of the vehicle structure, which are shown to be a flat output. The model of the car is a detailed nonlinear two-track vehicle with all degrees of freedom in the threedimensional space provided with redundant actuation. Each tire is equipped with two salient pole permanent magnet (PM-)synchronous machines for steering and driving. For the controller design, an overall model with a special differentialalgebraic structure is derived. The tire forces and the torques of the electrical machines are used as fictitious inputs. With this particular modeling it is possible to decouple the dynamic part of the model in the given coordinates at the highest relative degree. To get a consistent behavior it is necessary to account for some algebraic constraints, in this case given by the tire model and the torque equations of the PM-synchronous machines. It is possible to parameterize all constraints and coordinates of the drive units by the coordinates of the vehicle structure and its derivatives. All these parameterizations can be derived analytically. With an additional tracking controller the approach is robust against parameter variations and pulseshaped disturbances. The control of the electrical machines is integrated in the overall control law. By introducing additional coupling conditions it is possible to generate the desired torque for steering and driving by minimum current.
\end{abstract}

\section{INTRODUCTION}

Today, the control of vehicle dynamics is a frequently used method to improve the safety of vehicles and the comfort for passengers. With the slow transition from the combustion engine to the electrical motor new architectures and their control become a main point of interest. There are several contributions about vehicles with independent driving, braking and steering of each tire, e.g. [10]. Generalized forces are allocated to the longitudinal and lateral tire forces which depend on the slip, the friction coefficient and the normal tire force, thus slip is often the controlled parameter. Many articles focus on optimizing the distribution of the tire forces $[6],[1],[5]$. In most contributions the assumed input variables are the torques for driving and the steering angles. There are much less investigations that take the dynamics of the electrical machines into account like [3] and [9]. The control laws are often designed separate for several subsystems and couplings are regarded as disturbances.

This contribution presents the design of an overall control law in two sequential steps with differential geometric methods. The dynamics of the actuators and the steering as well as the couplings between several subsystems are taken into

J. Hoedt is coworker at the Department of Control Engineering and Mechatronics, Technische Universität Darmstadt, 64283 Darmstadt, Germany, jhoedt@iat.tu-darmstadt.de

U. Konigorski is head of the Department of Control Engineering and Mechatronics, Technische Universität Darmstadt, 64283 Darmstadt, Germany, ukonigorskieiat.tu-darmstadt.de account analytically. Section II presents the model of the car used for the controller design. Fictitious inputs are used to derive a differential-algebraic model in which the dynamic part is given in controller normal form. Section III presents the design of the decoupling controller which transforms the system in Brunovský normal form and the additional design of a tracking controller. The parameterization of the constraints by the vehicle structure coordinates and the introduction of additional coupling conditions to generate trajectories for minimum current are shown. Some simulation results are presented in section IV followed by the conclusion in section $\mathrm{V}$.

\section{MODELING}

The derivation of the vehicle's subsystes is known from many contributions. A short review of the essential model properties is given. For more detailed explanations, see e.g. [6]. See fig 1, 2 and table I for the explanation of substantial parameters.

\section{A. Horizontal dynamics}

The horizontal dynamics describe the behavior of the vehicle in the horizontal plane and there are three degrees of freedom, namely the longitudinal velocity $v_{\mathrm{x}}$, the lateral velocity $v_{\mathrm{y}}$, and the yaw rate $\dot{\psi}$. The corresponding equations are given by

$$
\begin{aligned}
\dot{x}_{\mathrm{h}} & =f_{\mathrm{h}}\left(x_{\mathrm{h}}\right)+B_{\mathrm{h}} u_{\mathrm{h}} \\
y_{\mathrm{h}} & =x_{\mathrm{h}}
\end{aligned}
$$

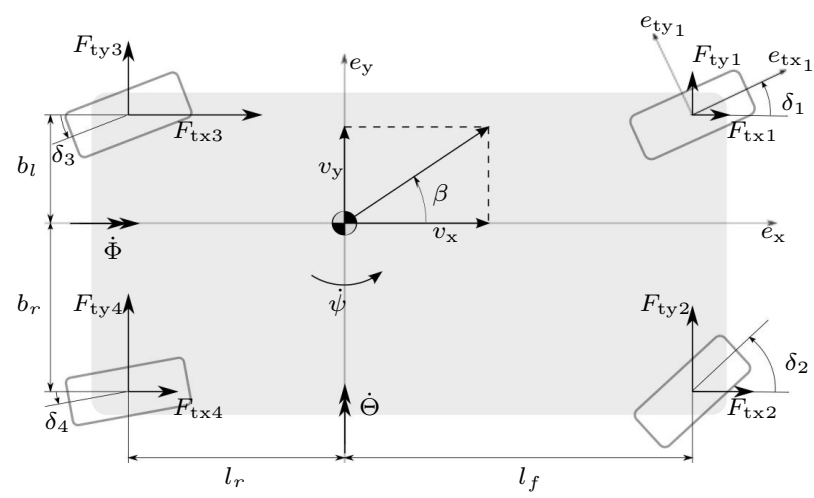

Fig. 1. Vehicle model in the horizontal plane 
with

$$
x_{\mathrm{h}}=\left[\begin{array}{c}
v_{\mathrm{x}} \\
v_{\mathrm{y}} \\
\dot{\psi}
\end{array}\right], B_{\mathrm{h}}=\left[\begin{array}{ccc}
\frac{1}{m} & 0 & 0 \\
0 & \frac{1}{m} & 0 \\
0 & 0 & \frac{1}{J_{\mathrm{z}}}
\end{array}\right], u_{\mathrm{h}}=P F_{\mathrm{t}}, v_{\mathrm{t}}=P^{T} x_{\mathrm{h}}
$$

and

$$
\begin{aligned}
& F_{\mathrm{t}}=\left[\begin{array}{llllllll}
F_{\mathrm{tx} 1} & F_{\mathrm{ty} 1} & F_{\mathrm{tx} 2} & F_{\mathrm{ty} 2} & F_{\mathrm{tx} 3} & F_{\mathrm{ty} 3} & F_{\mathrm{tx} 4} & F_{\mathrm{ty} 4}
\end{array}\right]^{T}, \\
& v_{\mathrm{t}}=\left[\begin{array}{llllllll}
v_{\mathrm{tx} 1} & v_{\mathrm{ty} 1} & v_{\mathrm{tx} 2} & v_{\mathrm{ty} 2} & v_{\mathrm{tx} 3} & v_{\mathrm{ty} 3} & v_{\mathrm{tx} 4} & v_{\mathrm{ty} 4}
\end{array}\right]^{T} .
\end{aligned}
$$

The Matrix $P$ has rank three, thus the right inverse exists and the tire forces $F_{\mathrm{t}}$ can be written as

$$
F_{\mathrm{t}}=P^{+} u_{\mathrm{h}}+P^{\perp} \rho_{\mathrm{h}}
$$

with $P^{+}$the right inverse and $P^{\perp}$ a kernel of $P$. The parameters $\rho_{\mathrm{h}}$ have no direct influence on the motion of the vehicle but they can change the distribution of the tire forces. For the basic controller design these parameters are set to zero. $v_{\mathrm{t}}$ describes the velocities of the tires in terms of $x_{h}$. The effect $u_{\mathrm{h}}$ of the tire forces on the center of mass is used as fictitious input. Note that the horizontal dynamics are in controller normal form because of this modeling.

\section{B. Vertical dynamics}

For the sake of clarity, a linearized model of the vertical dynamics is used. The vertical dynamics are given by

$$
\begin{aligned}
& \dot{x}_{\mathrm{v}}=A_{\mathrm{v}} x_{\mathrm{v}}+B_{\mathrm{v}}\left(S u_{z}+K u_{\mathrm{h}}\right) \\
& F_{\mathrm{z}}=N x_{\mathrm{v}}+u_{\mathrm{z}}+F_{\mathrm{z}_{\mathrm{p} 1}} \\
& y_{\mathrm{v}}=C_{\mathrm{v}} x_{\mathrm{v}}
\end{aligned}
$$

with

$$
x_{\mathrm{v}}=\left[\begin{array}{c}
\Delta z \\
\Delta \dot{z} \\
\Phi \\
\dot{\Phi} \\
\Theta \\
\dot{\Theta}
\end{array}\right] \quad B_{\mathrm{v}}=\left[\begin{array}{ccc}
0 & 0 & 0 \\
\frac{1}{m} & 0 & 0 \\
0 & 0 & 0 \\
0 & \frac{1}{J_{x}} & 0 \\
0 & 0 & 0 \\
0 & 0 & \frac{1}{J_{y}}
\end{array}\right], K=\left[\begin{array}{ccc}
0 & 0 & 0 \\
0 & s & 0 \\
-s & 0 & 0
\end{array}\right] \text {. }
$$

The tire normal force $F_{\mathrm{z}}$ depends on the vertical dynamics coordinates and $F_{\mathrm{z}_{\mathrm{p} 1}}$ induced by the prestressed springs of the passive part of the suspension. The inputs $u_{\mathrm{z} i}$ are forces that can be applied to the chassis by an active suspension. The term $K u_{\mathrm{h}}$ specifies the interconnection to the horizontal dynamics. $u_{\mathrm{v}}=S u_{\mathrm{z}}$ describes the effect of the inputs on the

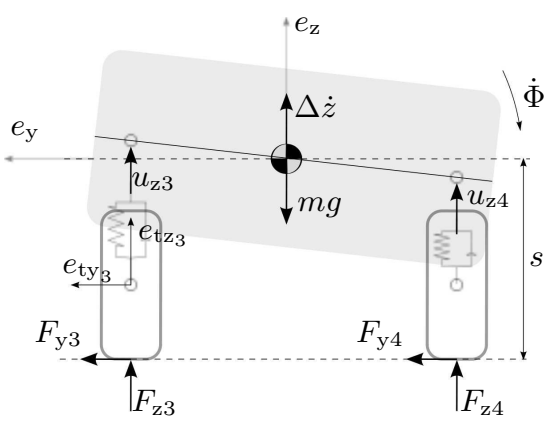

Fig. 2. Vertical view of the vehicle model center of mass. Because $S$ has rank three it is possible to state

$$
u_{\mathrm{z}}=S^{+} u_{\mathrm{v}}+S^{\perp} \rho_{\mathrm{v}},
$$

with $S^{+}$the right inverse and $S^{\perp}$ a kernel of $S$. The parameter $\rho_{\mathrm{v}}$ influences the distribution to the tire normal forces and is set to zero here. With $u_{\mathrm{v}}$ as input, the vertical dynamics are in controller normal form, too. The output $y_{\mathrm{v}}$ is described by the three displacements $\Delta z, \Phi$ and $\Theta$.

\section{Tire and drive dynamics}

Each tire has two degrees of freedom, namely the rotation around the $\mathrm{z}$-axis and $\mathrm{y}$-axis of the tire coordinate $\left(e_{\mathrm{tx}_{i}}, e_{\mathrm{ty}_{i}}, e_{\mathrm{tz}_{i}}\right)$ for steering $\delta_{i}$ and driving $\omega_{i}$. In a simplified model of the steering kinematics it is assumed that there is no camber, caster or toe. The behavior can be described by

$$
\begin{aligned}
J_{\mathrm{ty}} \dot{\omega}_{i} & =M_{\mathrm{y} i}-r_{\mathrm{dyn}}\left(F_{\mathrm{tx} i} \cos \left(\delta_{i}\right)+F_{\mathrm{ty} i} \sin \left(\delta_{i}\right)\right) \\
J_{\mathrm{tz}} \ddot{\delta}_{i} & =M_{\mathrm{z} i} i=1, \ldots, 4 .
\end{aligned}
$$

$M_{\mathrm{y} i}$ and $M_{\mathrm{z} i}$ are the torques generated by the PMsynchronous machines. Their dynamic equations are given in coordinates of field-orientated currents $I_{\mathrm{d}}$ and $I_{\mathrm{q}}$ and it is assumed that the input voltages $U_{\mathrm{q}}, U_{\mathrm{d}}$ can be exactly provided. The electrical machines are described by

$$
\begin{aligned}
& L_{\mathrm{q}_{\mathrm{j} i}} \dot{I}_{\mathrm{q}_{\mathrm{j} i}}=U_{\mathrm{q}_{\mathrm{j} i}}-R_{\mathrm{j} i} I_{\mathrm{q}_{\mathrm{j} i}}-Z_{\mathrm{p}_{\mathrm{j} i}} \Omega_{\mathrm{j} i}\left(\Psi_{\mathrm{PM}_{\mathrm{j} i}}+L_{\mathrm{d}_{\mathrm{j} i}} I_{\mathrm{d}_{\mathrm{j} i}}\right) \\
& L_{\mathrm{d}_{\mathrm{j} i}} \dot{I}_{\mathrm{d}_{\mathrm{j} i}}=U_{\mathrm{d}_{\mathrm{j} i}}+R_{\mathrm{j} i} I_{\mathrm{d}_{\mathrm{j} i}}-Z_{\mathrm{p}_{\mathrm{j} i}} \Omega_{\mathrm{j} i} L_{\mathrm{q}_{\mathrm{j} i}} I_{\mathrm{q}_{\mathrm{j} i}}, \\
& i=1, \ldots, 4, \quad \mathrm{j}=\mathrm{y}, \mathrm{z}, \quad \Omega_{\mathrm{y} i}=\omega_{i}, \quad \Omega_{\mathrm{z} i}=\dot{\delta}_{i},
\end{aligned}
$$

see table I for substantial parameters. In fact, the dynamics of the tires and the drives are highly coupled through the torque equations of the electric machines. To derive a simple dynamic structure, the torque equations for the dynamics are not considered and the torques are modeled as fictitious inputs. This kind of modeling makes sure that the tire and drive dynamics have a simple dynamic structure and a special form as well as the other dynamic subsystems. Written as system of first order differential equations the nonlinear controller normal form

$$
\begin{aligned}
& \dot{x}_{\mathrm{t} i}=f_{\mathrm{t} i}\left(x_{\mathrm{t} i}\right)+G_{\mathrm{t} i} u_{\mathrm{t} i}+k_{\mathrm{t} i}\left(x_{\mathrm{t} i}\right) u_{\mathrm{h}} \\
& y_{\mathrm{t} i}=C_{\mathrm{t} i} x_{\mathrm{t} i},
\end{aligned}
$$

with

$$
\begin{aligned}
& x_{\mathrm{t} i}=\left[\begin{array}{lllllll}
I_{\mathrm{q}_{\mathrm{y} i}} & I_{\mathrm{q} i} & \omega_{i} & I_{\mathrm{q} i} & I_{\mathrm{q}_{\mathrm{y} i}} & \delta_{i} & \dot{\delta}_{i}
\end{array}\right]^{T}, \\
& u_{\mathrm{t} i}=\left[\begin{array}{llllll}
U_{\mathrm{q} i} & U_{\mathrm{d}_{\mathrm{y} i}} & M_{\mathrm{y} i} & U_{\mathrm{q}_{\mathrm{z} i}} & U_{\mathrm{d}_{\mathrm{z} i}} & M_{\mathrm{z} i}
\end{array}\right]^{T} \text {, } \\
& C_{\mathrm{t} i}=\left[\begin{array}{ll}
E_{6} & 0_{6 \times 1}
\end{array}\right] \text {. }
\end{aligned}
$$

of the tire and drive dynamics is obtained. The matrix $E_{6}$ is the six dimensional identity. In (8), the tire forces from (6) are translated to $u_{\mathrm{h}}$ with $P^{+}$.

\section{Constraints}

Several fictitious inputs are used to derive all dynamic subsystems in controller normal form. These fictitious inputs 
can not be chosen independently, they must satisfy some constraints. The torques in (8) must satisfy the torque equations for the electrical machines (7), which are given by

$$
\begin{aligned}
& 0=M_{\mathrm{j} i}-\frac{3}{2} Z_{\mathrm{p}_{\mathrm{j} i}}\left(\Psi_{\mathrm{PM}_{\mathrm{j} i}} I_{\mathrm{q}_{\mathrm{j} i}}+\left(L_{\mathrm{d}_{\mathrm{j} i}}-L_{\mathrm{q}_{\mathrm{j} i}}\right) I_{\mathrm{q}_{\mathrm{j} i}} I_{\mathrm{d}_{\mathrm{j} i}}\right) \\
& i=1, \ldots, 4, \quad \mathrm{j}=\mathrm{y}, \mathrm{z}, \quad \Omega_{\mathrm{y} i}=\omega_{i}, \quad \Omega_{\mathrm{z} i}=\dot{\delta}_{i} .
\end{aligned}
$$

In standard approaches the field generating current $I_{\mathrm{d}}$ is often set to zero. In the case of a machine afflicted with reluctance there is another way to use this degree of freedom. In the presented model the electrical machines are direct drives. Therefore the speed is relatively small and iron loss is dominating. By minimizing the current that generates the desired torque, the iron loss is minimized too. This minimization is called maximum torque per ampere (MTPA) and is well known from literature, e.g. [7]. The solution of the minimization for the motor mode $\left(I_{\mathrm{q} i}>0\right)$ gives the current $I_{\mathrm{d}_{i}}$ as a function of $I_{\mathrm{q}_{i}}$.

$$
0=I_{\mathrm{d}_{\mathrm{j} i}}+\frac{\Psi_{\mathrm{PM}_{\mathrm{j} i}}}{2\left(L_{\mathrm{d}_{\mathrm{j} i}}-L_{\mathrm{q}_{\mathrm{j} i}}\right)}+\sqrt{\left(\frac{\Psi_{\mathrm{PM}_{\mathrm{j} i}}}{2\left(L_{\mathrm{d}_{\mathrm{j} i}}-L_{\mathrm{q}_{\mathrm{j} i}}\right)}\right)^{2}+I_{\mathrm{q}_{\mathrm{j} i}}^{2}}
$$

This equation is used as additional artificial constraint or rather coupling condition. For $u_{\mathrm{h}}$ the constraints are given by the tire model, which describes the generation of the tire forces phenomenologically. The used tire model is a simplified, analytic invertible version of the Magic Formula Tire Model, taken from [6]. The longitudinal and side slip

$$
\begin{aligned}
s_{\mathrm{x} i} & =\frac{r_{\mathrm{dyn}} \omega_{i} \cos \left(\delta_{i}\right)-v_{\mathrm{tx} i}}{\sqrt{v_{\mathrm{tx} i}^{2}+v_{\mathrm{ty} i}^{2}}}=f_{\mathrm{s}_{\mathrm{x}} i}\left(\omega_{i}, \delta_{i}, x_{\mathrm{h}}\right), \\
s_{\mathrm{y} i} & =\frac{r_{\mathrm{dyn}} \omega_{i} \sin \left(\delta_{i}\right)-v_{\mathrm{ty} i}}{\sqrt{v_{\mathrm{tx} i}^{2}+v_{\mathrm{ty} i}^{2}}}=f_{\mathrm{s}_{\mathrm{y}} i}\left(\omega_{i}, \delta_{i}, x_{\mathrm{h}}\right)
\end{aligned}
$$

are functions of $\omega_{i}, \delta_{i}$ and the coordinates of the horizontal dynamics. The maximum transferable force

$$
F_{\max _{i}}=\mu_{i} F_{\mathrm{z} i}\left(1+k_{\mathrm{Fz}} \frac{F_{\mathrm{z} 0}-F_{\mathrm{z} i}}{F_{\mathrm{z} 0}}\right)=f_{\max _{i}}\left(x_{\mathrm{v}}, u_{\mathrm{z}}\right)
$$

at each tire declining depends on $F_{\mathrm{z} i}$ and therefore is a function of $x_{\mathrm{v}}$ and $u_{\mathrm{z}}$ or $u_{\mathrm{v}}$, respectively. With the slip and the maximal transferable force the tire forces are given by

$$
\begin{aligned}
& 0=F_{\mathrm{tj} i}-F_{\max _{i}} \cdot \ldots \\
& \ldots \sin \left(C_{i} \arctan \left(\frac{B_{i}}{\mu_{i}} \sqrt{s_{\mathrm{x} i}^{2}+s_{\mathrm{y} i}^{2}}\right)\right) \frac{s_{\mathrm{j} i}}{\sqrt{s_{\mathrm{x} i}^{2}+s_{\mathrm{y} i}^{2}}}
\end{aligned}
$$

with $\mathrm{j}=\mathrm{x}, \mathrm{y}$ at the $i$-th tire.

The constraints for the overall model are given by eight torque equations (10), which are force-constraints, depending on fictitious inputs and differential variables of the drives, eight coupling conditions (11) for differential variables, and eight equations of the form (14), which depend on fictitious inputs, real inputs, and differential variables of all dynamic subsystems.

\section{E. Model for the overall system}

Now it is possible to derive an overall model. With the vector

$$
x=\left[\begin{array}{llllll}
x_{\mathrm{h}}^{T} & x_{\mathrm{t} 1}^{T} & x_{\mathrm{t} 2}^{T} & x_{\mathrm{t} 3}^{T} & x_{\mathrm{t} 4}^{T} & x_{\mathrm{v}}^{T}
\end{array}\right]^{T}
$$

of the differential variables and the output

$$
y=\left[\begin{array}{llllll}
y_{\mathrm{h}}^{T} & y_{\mathrm{t} 1}^{T} & y_{\mathrm{t} 2}^{T} & y_{\mathrm{t} 3}^{T} & y_{\mathrm{t} 4}^{T} & y_{\mathrm{v}}^{T}
\end{array}\right]^{T}
$$

the system equations are

$$
\begin{aligned}
\dot{x} & =f(x)+g(x) u \\
0 & =\hat{q}(x, u) \\
y & =C x .
\end{aligned}
$$

The drift and input vector are given by

$$
\begin{array}{lllllll}
f(x) & =\left[\begin{array}{llllll}
f_{\mathrm{h}}^{T} & f_{\mathrm{t} 1}^{T} & f_{\mathrm{t} 2}^{T} & f_{\mathrm{t} 3}^{T} & f_{\mathrm{t} 4}^{T} & \left(A_{\mathrm{v}} x_{\mathrm{v}}\right)^{T}
\end{array}\right]^{T}, \\
u & =\left[\begin{array}{llllll}
u_{\mathrm{h}}^{T} & u_{\mathrm{t} 1}^{T} & u_{\mathrm{t} 2}^{T} & u_{\mathrm{t} 3}^{T} & u_{\mathrm{t} 4}^{T} & u_{\mathrm{v}}^{T}
\end{array}\right] .
\end{array}
$$

This is a standard semi-explicit form for descriptor systems. For the described system the dimension of $x$ is $n=37$, and with the transformed inputs $u_{\mathrm{h}}$ and $u_{\mathrm{v}}$ the property $\operatorname{dim}(u)=\operatorname{dim}(y)=30$ holds. With the voltages of the electrical machines as well as the transformed active suspension forces there are 20 real inputs and the torques plus the transformed tire forces result in 10 fictitious inputs. The output $y$ is intuitive and includes all interesting system variables. The algebraic equation of (17) contains all constraints from section II-D. For later considerations the outputs

$$
\begin{aligned}
& y_{\mathrm{p} 1}=\left[\begin{array}{ll}
y_{\mathrm{h}}^{T} & y_{\mathrm{v}}^{T}
\end{array}\right]^{T}, \\
& y_{\mathrm{p} 2}=\left[\begin{array}{llll}
y_{\mathrm{t} 1}^{T} & y_{\mathrm{t} 2}^{T} & y_{\mathrm{t} 3}^{T} & y_{\mathrm{t} 4}^{T}
\end{array}\right]^{T}
\end{aligned}
$$

are defined.

\section{Controller Design}

The system was constructed in a way, that all dynamic subsystems are in controller normal form. In fact the overall system equations can be written in the form

$$
\begin{aligned}
\dot{\xi}_{i}^{j} & =\xi_{i}^{j+1} \\
\dot{\xi}_{i}^{\kappa_{i}} & =\alpha_{i}\left(\bar{\xi}_{1}^{\kappa_{1}-1}, \ldots, \bar{\xi}_{p}^{\kappa_{p}-1}\right)+\beta_{i}^{T}\left(\bar{\xi}_{1}^{\kappa_{1}-1}, \ldots, \bar{\xi}_{p}^{\kappa_{p}-1}\right) u \\
0 & =q\left(\bar{\xi}_{1}^{\kappa_{1}-1}, \ldots, \bar{\xi}_{p}^{\kappa_{p}-1}, u\right) \\
\bar{\xi}_{i}^{\kappa_{i}} & =\left(\xi_{i}^{1}, \xi_{i}^{2}, \ldots, \xi_{i}^{\kappa_{i}}\right), i=1,2, \ldots, p ; \quad j=1,2 \ldots, \kappa_{i}-1
\end{aligned}
$$

with $\xi^{1}=\left(\xi_{1}^{1}, \xi_{2}^{1}, \ldots, \xi_{p}^{1}\right)=y$ and $\operatorname{rank}(\beta)=\operatorname{dim}(u)=p$ holds, which clarifies the structure of the system equations.

\section{A. Decoupling and tracking control}

With the preliminaries from section III, it can be stated directly that $y$ is a flat output for the dynamic part of (17) disregarding the constraints. In the $\kappa_{i}$-th time derivative

$$
y^{(\kappa)}=\hat{\alpha}(x)+\hat{\beta}(x) u
$$


of the elements $y_{i}$ of $y$ the input appears explicit. Note, this is the same as just taking the equations with the highest derivatives $\xi_{i}^{\kappa_{i}}$ from (20). Thus, $y^{(\kappa)}$ is the vector with the highest derivatives $\kappa_{i}$ of $y_{i}$. The decoupling matrix $\hat{\beta}(x)$ is nonsingular, meaning that $\kappa=\left\{\kappa_{1}, \ldots, \kappa_{p}\right\}$ is the vector relative degree of the output $y$. Due to modeling the tire forces and torques as fictitious inputs, no complicated transformation into flat coordinates is necessary and $\sum_{i=1}^{q} \kappa_{i}=$ $\operatorname{dim}(x)$ holds. The feedback

$$
u=\hat{\beta}(x)^{-1}(-\hat{\alpha}(x)+v)
$$

transforms the dynamic part of (17) in Brunovský normal form. For more detailed explanations see, e.g. [2]. With the tracking controller

$$
v=y_{\mathrm{d}}^{(\kappa)}+Q_{k-1} e^{(\kappa-1)}+\ldots+Q_{1} \dot{e}+Q_{0} e
$$

and feasible matrices $Q_{i}$ the tracking error $e=y_{\mathrm{d}}-y$ is asymptotically stable where $y_{\mathrm{d}}$ are the desired trajectories. The controller design is carried out fast and easily due to modeling the car as descriptor system with the dynamic part in controller normal form. This advantage is accomplished by the fact, that the elements of $y$ are not independent. This will be treated in the next section.

\section{B. Parameterizations}

In the described approach the tire forces and the motor torques are completely independent inputs. To receive a consistent behavior of the overall system the trajectories for tracking control can not be chosen independently. They must comply with the constraints of (17). The concept of parameterizing inputs, states, and other equations by certain outputs is well established for flat systems, see [2] for a elaborate description. By parameterizing the input $u$ by the output and its derivatives as

$$
u=\tilde{\beta}\left(y^{(\kappa-1)}, \ldots, y\right)^{-1}\left(y^{(\kappa)}-\tilde{\alpha}\left(y^{(\kappa-1)}, \ldots, y\right)\right),
$$

it is possible to parameterize the constraints

$$
0=\tilde{q}\left(y^{(\kappa)}, y^{(\kappa-1)}, \ldots, y\right)
$$

as well. This means the trajectories of the output and its derivatives have to satisfy the implicit system of equations (25) for a realistic behavior of the system (17). To find the dependencies, the structure of (25) is analysed. The sixteen currents of the drives appear purely algebraic in the sixteen torque equations and the coupling conditions. Furthermore

$$
\operatorname{rank}\left(\frac{\partial \tilde{q}}{\partial I}\right)=16
$$

of the Jacobian matrix with $I$ containing all currents is maximal. Thus, by the implicit function theorem the constraints can at least locally be written in the form

$$
\begin{aligned}
I & =\tilde{q}_{1}^{-1}\left(\tilde{y}, \ldots, \tilde{y}^{(\kappa)}\right) \\
0 & =\tilde{q}_{2}\left(\tilde{y}, \ldots, \tilde{y}^{(\kappa)}\right), \\
\tilde{y} & =\left[\begin{array}{lll}
y_{\mathrm{p} 1} & \omega^{T} & \delta^{T}
\end{array}\right]^{T}
\end{aligned}
$$

with $\omega=\left[\omega_{1}, \ldots, \omega_{4}\right]^{T}$ and $\delta=\left[\delta_{1}, \ldots, \delta_{4}\right]^{T}$. In this case, it is possible to find an analytical solution, shown by using the example of the $i$-th drive for steering. The coupling condition can be solved for $I_{\mathrm{d}_{i}}$ and inserted into the torque equation. With a little calculation the polynomial

$$
I_{\mathrm{q}_{z i}}^{4}+\frac{2 J_{\mathrm{tz}} \ddot{\delta}_{i} \Psi_{\mathrm{PM}_{z i}}}{3 Z_{\mathrm{p}_{z i}} \Delta L_{z i}^{2}} I_{\mathrm{q}_{z i}}-\left(\frac{2 J_{\mathrm{tz}} \ddot{\delta}_{z i}}{3 Z_{\mathrm{p}_{z i}} \Delta L_{z i}}\right)^{2}=0
$$

of forth order in $I_{\mathrm{q}}$ with $\Delta L_{z i}=L_{\mathrm{d}_{z i}}-L_{\mathrm{q}_{z i}}$ can be derived. The solutions of this equation can be analytically calculated with an arbitrary computer algebra system. Considering the assumption $I_{\mathrm{q} i}>0$ and the side condition of the optimization it is essential that $I_{\mathrm{d} i}<0$, and only one solution remains. This considerations also can be investigated for all other operation modes like generator mode of the machines or driving backwards. In every case there are now parameterizations

$$
\begin{aligned}
& I_{\mathrm{j}_{\mathrm{zi}}}=\tilde{p}_{\mathrm{I}_{\mathrm{j} 0} z i}\left(\ddot{\delta}_{i}\right), \\
& \dot{\mathrm{I}}_{\mathrm{j}_{z i}}=\tilde{p}_{\mathrm{I}_{\mathrm{j} 0} z i}\left(\ddot{\delta}_{i}, \delta_{i}^{(3)}\right), \quad i=1, \ldots, 4, \mathrm{j}=\mathrm{q}, \mathrm{d} .
\end{aligned}
$$

The remaining eight constraints $\tilde{q}_{2}$ consist of the tire model, where $\omega$ and $\delta$ don't occur differentially in the equations. The property

$$
\operatorname{rank}\left(\frac{\partial \tilde{q}_{2}\left(\tilde{y}, \ldots, \tilde{y}^{(\kappa)}\right)}{\partial[\omega, \delta]}\right)=\operatorname{dim}\left(\tilde{q}_{2}\left(\tilde{y}, \ldots, \tilde{y}^{(\kappa)}\right)\right)=8
$$

shows, that there is at least a local parameterization for $\omega$ and $\delta$ by $y_{\mathrm{p}_{1}}$ and its derivatives. The simplified Magic Tire Formula gives the possibility for an analytical solution. Inserting the parameterization

$$
u_{\mathrm{h}}=B_{\mathrm{h}}^{-1}\left(\dot{y}_{\mathrm{h}}-f_{\mathrm{h}}\left(y_{\mathrm{h}}\right)\right)
$$

into the second derivative of the output equation of (4) yields to the parameterization

$$
u_{\mathrm{v}}=p_{\mathrm{v}}\left(y_{v}, \dot{y}_{v}, \ddot{y}_{v}, y_{\mathrm{h}}, \dot{y}_{\mathrm{h}}\right)
$$

which means, (13) can now also be written as function of $y_{\mathrm{h}}$, $y_{\mathrm{v}}$, and its derivatives and with (27) and (3) the tire forces can be written as function of $y_{\mathrm{h}}$ and its derivatives. Inverting the terms (14) results in

$$
s_{\mathrm{j} i}=\frac{\mu_{i}}{B_{i}} \tan \left(\frac{1}{C_{i}} \arcsin \left(\frac{\sqrt{F_{\mathrm{tx} i}^{2}+F_{\mathrm{ty} i}^{2}}}{F_{\max _{i}}}\right)\right) \frac{F_{\mathrm{tj} i}}{\sqrt{F_{\mathrm{tx} i}^{2}+F_{\mathrm{ty} i}^{2}}},
$$

with $i=1, \ldots, 4 ; j \in x, y$ which is therefore also a function of $y_{\mathrm{h}}, y_{\mathrm{v}}$ and its derivatives. By inverting (12) and inserting all previous parameterizations the terms

$$
\begin{aligned}
& \omega_{i}=p_{\omega_{i}}\left(y_{\mathrm{h}}, \dot{y}_{\mathrm{h}}, y_{\mathrm{v}}, \dot{y}_{\mathrm{v}}, \ddot{y}_{\mathrm{v}}\right), \\
& \delta_{i}=p_{\delta_{i}}\left(y_{\mathrm{h}}, \dot{y}_{\mathrm{h}}, y_{\mathrm{v}}, \dot{y}_{\mathrm{v}}, \ddot{y}_{\mathrm{v}}\right),
\end{aligned}
$$

hold, meaning $\omega$ and $\delta$ and their derivatives are completely parameterized by the coordinates of the vehicle structure and its derivatives. With (26) it is possible to get parameterizations

$$
I_{\mathrm{j}_{\mathrm{ki}}}=p_{\mathrm{I}_{\mathrm{j}_{\mathrm{ki}}}}\left(y_{\mathrm{h}}, \dot{y}_{\mathrm{h}}, \ldots, y_{\mathrm{h}}^{(3)}, y_{\mathrm{v}}, \dot{y}_{\mathrm{v}}, \ldots, y_{\mathrm{v}}^{(4)}\right) \text {, }
$$


with $\mathrm{j}=\mathrm{q}, \mathrm{d} ; k=\mathrm{y}, \mathrm{z} ; i=1, \ldots, 4$. for the currents. At this point the parameterization is concluded. All parameterizations are purely algebraic and no integration is necessary. The whole system can be parameterized by the coordinates of the vehicle structure which proofs that these coordinates are a flat output of the system in minimal coordinates. In other words, with (30), (31), and (22), a Lie-Bäcklund equivalence to a trivial system of six integrator chains can be constructed. For the designed tracking controller it is necessary to calculate the first derivative of the currents, meaning that the fourth derivative of $y_{\mathrm{h}}$ and the fifth derivative of $y_{\mathrm{v}}$ is needed.

\section{Trajectory generation}

There are many possibilities to translate the driver's input to trajectories for the horizontal dynamic. It depends on how the driving should "feel". Companies and researchers spend a lot work on this question. The following approach is just a simple proposal with the purpose to be not too complicated. In further work limitations in traction [4] and structural constraints will be integrated into the reference trajectory generation. For a real time implementation of this controller design it is necessary to generate trajectories from the driver input nearly without delay. A change of the throttle position can be interpreted as a change of the desired velocity in driving direction, namely $v_{x}$. The difference between old and new position can be interpreted as a step $u_{\mathrm{v}_{\mathrm{d}}}$ which is used as input for a linear model

$$
v_{\mathrm{x}_{\mathrm{d}}}^{(4)}=-\alpha_{\mathrm{x}_{3}} v_{\mathrm{x}_{\mathrm{d}}}^{(3)}-\alpha_{\mathrm{x}_{2}} \ddot{v}_{\mathrm{x}_{\mathrm{d}}}-\alpha_{\mathrm{x}_{1}} \dot{v}_{\mathrm{x}_{\mathrm{d}}}-\alpha_{\mathrm{x}_{0}} v_{\mathrm{x}_{\mathrm{d}}}+\kappa_{x} u_{\mathrm{v}_{\mathrm{d}}} \text {. }
$$

The parameters of (32) should be chosen in such a way that the generated trajectories are asymptotically stable. The same approach can be used for the yaw rate. A steering angle can be interpreted as a desired yaw rate. The gain should depend on the velocity of the vehicle. For the velocity $v_{\mathrm{y}}$ orthogonal to the driving direction, several approaches could be used. One possibility is to set $v_{\mathrm{y}}$ and its derivatives to zero. This would be the same as making the slip angle $\beta$ to zero. Apparently the coordinates of the vertical dynamics are a part of the flat system output and contribute a fundamental part to system dynamics. Its trajectories and its derivatives are set to zero in this approach. If there are no actuators for the vertical dynamic and the suspension is therefore passive, the controller design is also possible. The decoupling and tracking control is then carried out without the vertical dynamics, which can be treated as internal dynamics of the system in input-output normal form with the output

$$
\tilde{y}=\left[\begin{array}{ll}
y_{\mathrm{h}} & y_{\mathrm{p} 2}
\end{array}\right]^{T} \text {. }
$$

To generate the needed trajectories for the parameterizations the internal dynamics will be integrated in the reference trajectory generation. This is possible because the eigenvalues eig $\left(A_{\mathrm{v}}\right)$ of the internal dynamics are stable with a reasonable suspension. The desired trajectories are calculated by

$$
\dot{x}_{\mathrm{v}_{\mathrm{d}}}=A_{\mathrm{v}} x_{\mathrm{v}_{\mathrm{d}}}+B_{\mathrm{v}} K B_{\mathrm{h}}^{-1}\left(\dot{y}_{\mathrm{h}_{\mathrm{d}}}-f_{\mathrm{h}}\left(y_{\mathrm{h}_{\mathrm{d}}}\right)\right) \text {. }
$$

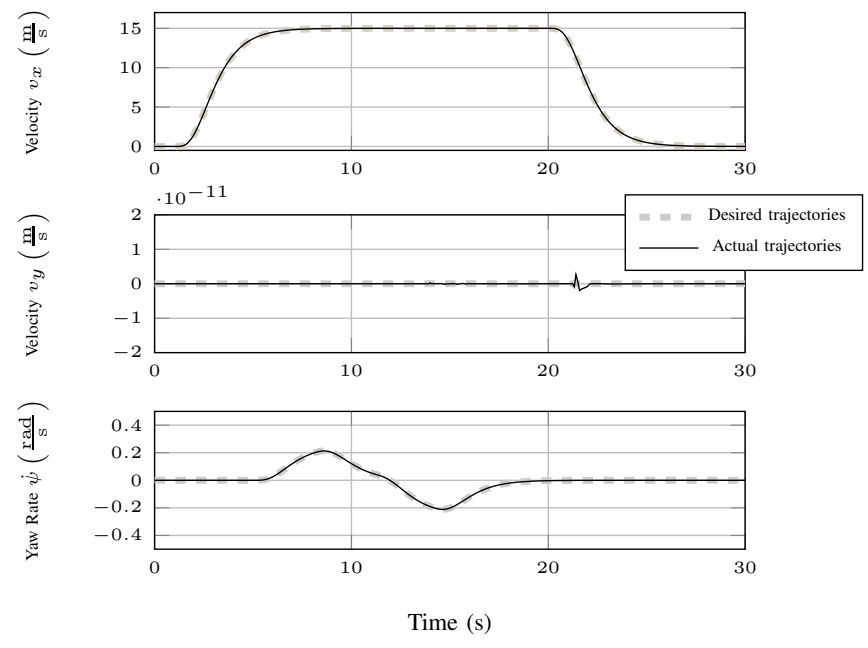

Fig. 3. Movement in the horizontal plane

In this case $y_{\mathrm{p} 1}$ is not a flat output but a parameterizing output. With the trajectories from models like (32) all other desired trajectories for the tracking controller (23) can be generated with (30) and (31).

\section{Simulation}

This section shows some simulation results for the described controller design. The controller has also been verified with a simulation model that only has real inputs. The parameters like car mass, suspension, etc. are taken from [6]. The parameters of the electrical machines are slightly adopted from [8] to show the effect of distinctive reluctance. Some key data are given in table I. Fig. 3 shows the driven maneuver in the horizontal plane. The driver accelerates to approximately $50 \mathrm{~km} / \mathrm{h}$, drives two curves and then decelerates. The car follows exactly the desired trajectories. The corresponding steering angles are shown in fig. 4. Again the desired trajectories and the steering angles of the car exactly conform. Obviously, the steering angles at the front and at rear must be in the opposite direction to achieve a velocity of zero in lateral direction. The angles at one axis are similar but not identical. Fig. 5 shows the current $I_{1_{1}}=\sqrt{I_{\mathrm{q}_{\mathrm{y} 1}}^{2}+I_{\mathrm{d}_{\mathrm{y} 1}}^{2}}$ of the electrical machine for driving of the left front tire. In peak times the current of the MTPA-control is more than fifteen percent lower than with the current trajectories of the $I_{d}=0$-approach. Fig. 6

TABLE I

PARAMETERS OF THE CAR AND ELECTRICAL MACHINES

\begin{tabular}{ccc}
\hline Vehicle mass & $m$ & $1250 \mathrm{Kg}$ \\
Moments of inertia & $J_{\mathrm{x}}$ & $430 \mathrm{~kg} \mathrm{~m}^{2}$ \\
& $J_{\mathrm{y}}$ & $2000 \mathrm{~kg} \mathrm{~m}^{2}$ \\
& $J_{\mathrm{z}}$ & $2200 \mathrm{~kg} \mathrm{~m}^{2}$ \\
& $J_{\omega}$ & $1.1 \mathrm{~kg} \mathrm{~m}^{2}$ \\
\hline Number of pole pairs & $Z_{\mathrm{p}_{\mathrm{y} i}}$ & 8 \\
Resistance & $R_{\mathrm{p}_{\mathrm{y} i}}$ & $160 \mathrm{~m} \Omega$ \\
Inductance d-axis & $L_{\mathrm{d}_{\mathrm{y} i}}$ & $2 \mathrm{mH}$ \\
Inductance q-axis & $L_{\mathrm{q}_{\mathrm{y} i}}$ & $3.2 \mathrm{mH}$ \\
PM-flux linkage & $\Psi_{\mathrm{PM}_{\mathrm{y} i}}$ & $318 \mathrm{mWb}$ \\
\hline
\end{tabular}


shows, that the accuracy of the control is raised if the vertical dynamics are considered as internal dynamics in case of a passive suspension. In case of treating the vertical dynamics as disturbance, the tire normal force and the maximum transferable force are set to constant values. This can be critical when the vehicle reaches its dynamic limits.

\section{CONCLUSION AND FUTURE WORK}

A new approach for the controller design for electric cars in x-by-wire structure was shown. With the help of a differential-algebraic model it was possible to achieve a differential parameterization. An overall control law was presented with integrated control of the electrical machines. By introducing the solution of the MTPA as additional coupling condition, it was possible to achieve a parameterization with minimum current for the desired torque. For the controller design it is not necessary to distinguish between real and fictitious inputs. It is relatively independent of the accuracy of the used subsystems. The accuracy can be adopted as necessary. The modeling is a essential part of the controller design, because the model is constructed to make the controller design fast and easy. In this application, the presented controller design has a great potential to achieve further goals. In the simplified tire model $F_{\max _{i}} \geq \sqrt{F_{\mathrm{tx} i}^{2}+F_{\mathrm{ty} i}^{2}}$ holds. This assumption can be substantial different to real circumstances and is treated in [4] by estimating the difference between the model and the real tire forces and by taking uncertainties and limits in the traction between road and tire into account by several differential parameterizations. Other future goals are e.g. optimizing the vertical dynamics trajectories for minimum change of the wheel load. The non-used parameters $\rho_{\mathrm{h}}$ can be used to adapt the controller design for other actor configurations and to accumulate actor failures, to achieve failure tolerance and a minimal actor configuration. The controller design is the application of a general concept for tracking control of descriptor systems,
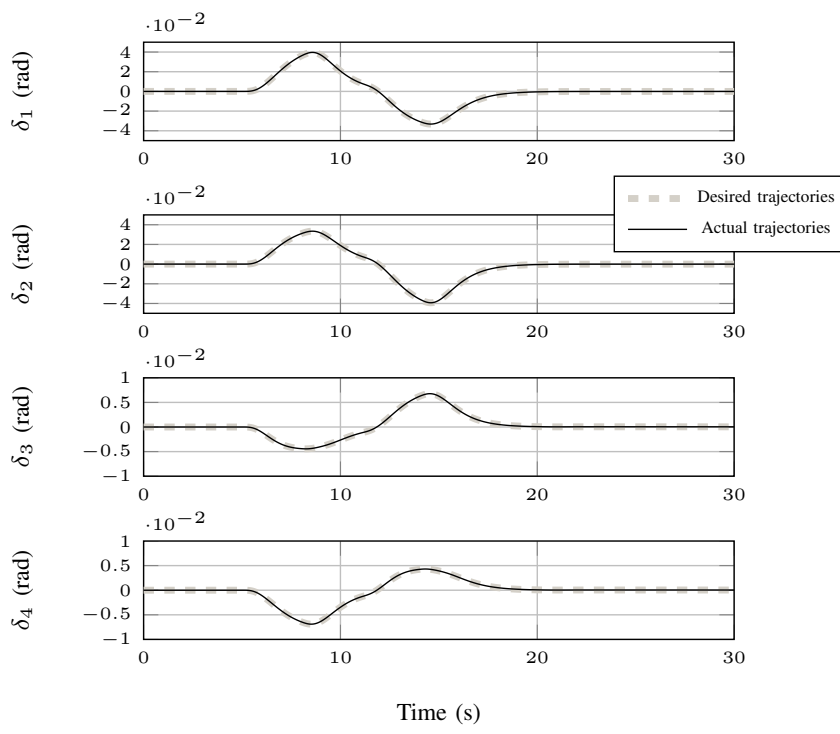

Fig. 4. Corresponding steering angles

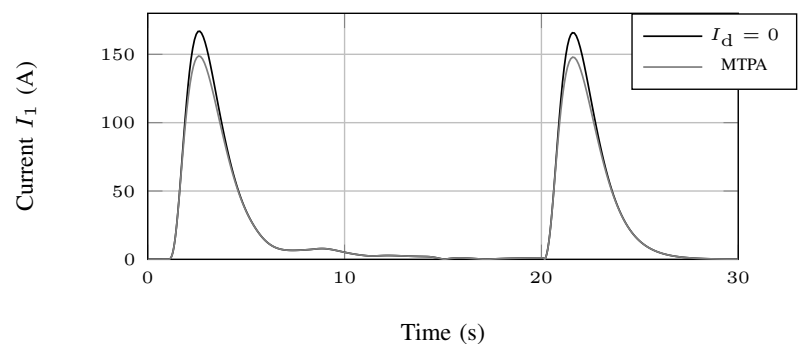

Fig. 5. Current $I_{1}$ with MTPA and $I_{d}=0$ control

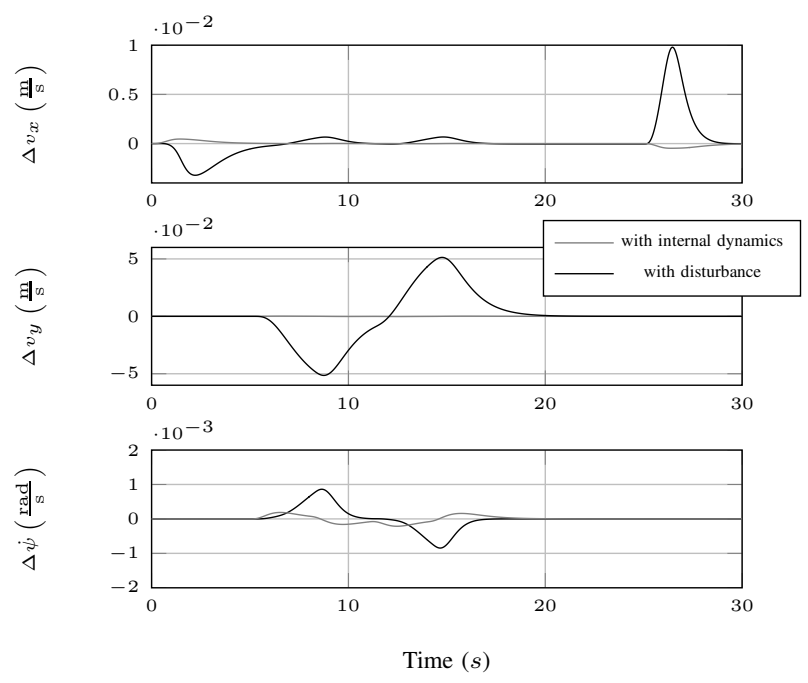

Fig. 6. Error with vertical dynamics as disturbance and internal dynamics

which will be elaborated in further work too. A special thanks goes to the reviewers for their competent comments.

\section{REFERENCES}

[1] Javad Ahmadi, Ali Khaki Sedigh, and Mansour Kabganian. Adaptive vehicle lateral-plane motion control using optimal tire friction forces with saturation limits consideration. IEEE Transactions on vehicular technology, 58:4098 - 4107, 2009.

[2] F. Antritter. Tracking Controller Design for Nonlinear Dynamics using Differential Parameterizations. $\mathrm{PhD}$ thesis, Universität der Bundeswehr München, 2007.

[3] Avesta Goodarzi and Ebrahim Esmailzadeh. Design of a vdc system for all-wheel independent drive vehicles. In IEEE/ASME Transactions On Mechatronics, 2007.

[4] Jens Hoedt and Ulrich Konigorski. Integrierte Fahrdynamikregelung mittels differentieller Parametrierungen. In AUTOREG 2011 Steuerung und Regelung von Fahrzeugen und Motoren, 2011.

[5] C. Knobel, A. Pruckner, and T. Bünte. Optimized force allocation a general approach to control and to investigate the motion of overactuated vehicles. In 4th IFAC-Symposium on Mechatronic Systems, 2006.

[6] Ralf Orend. Integrierte Fahrdynamikregelung mit Einzelradaktorik. $\mathrm{PhD}$ thesis, Universität Erlangen, 2006.

[7] Dierk Schröder. Elektrische Antriebe - Regelung von Antriebssystemen. Spinger, 2009.

[8] Oskar Wallmark. Control of Permanent-Magnet Synchronous Machines in Automotive Applications. PhD thesis, Chalmer University of Technology, 2006.

[9] Junmin Wang and Raul G. Longoria. Coordinated and reconfigurable dynamics control. IEEE Transactions On Control Systems Technology, 17:723 -732, May 2009.

[10] Lu Xiong and Zhuoping Yu. Control allocation of vehicle dynamics control for a 4 in-wheel-motored ev. In 2nd International Conference on Power Electronics and Intelligent Transportation System, 2009. 